OC 2.1

\section{Cervical Discs and Ozonucleolysis}

\section{Umair Rashid, A. Hameed}

Punjab Institute of Neuro Sciences, Lahore, Pakistan. E-mail:dr_umairch@yahoo.com

Background: We report our experience with ozonucleolysis between June 2005 to November 2016 with 4000 patients affected by pain cervical region (Bracehalga) due to disc herniation including of fibromylegia. Methods: All these cases treated by intradiscal, or paravertabral oxygen - ozone injections. Patients age between 20 to 70 years underwent percutenous ozoneuclealysis. The procedure done under the angiofioursocopy with full aspectic technique. The ozone generator, essential component placed close to the patients. Simple $23 \mathrm{G}$ needle to $22 \mathrm{G}$ spinal needle, (quincke type point) were used to inject ozone under fluoroscopy. No premedication or anesthesia were given and the procedures were performed at an outpatient facility with short hospital stay after the treatment. Results: Among 4000 patients 2000 patients were followed up for 5 months, $50 \%$ of the treated patients showed complete recovery with disappearance of symptoms. $25 \%$ of cases complaint of occasional episodes of pain neck and arms but no limitations of occupational activities $-15 \%$ of the cases showed in sufficient improvement $-5 \%$ cases no improvement and went for surgery $10 \%$ of the cases never turned up after the first visit. Most of these patient had no FDA surgical indication. The patients who failed to benefit from ozonucleolysis underwent surgery. In all these cases, the previous $\mathrm{O} 2 \mathrm{O} 3$ gas therapy had no negative effects on the surgical procedure. Conclusions: In our experience, Ozone Gas Therapy in treatment of cervical herniated disc has revolutionized the percutenous approach to nerve root disease making it safer cheaper and easier to repeat than treatments currently in use.

OC 2.2

Microwave Ablation for Hepatocellular Carcinoma-Initial Experience at a Large Tertiary Care Center in Riyadh, Kingdom of Saudi Arabia

\section{Turki M. Hamdi, Shahbaz Ahmed Qazi, Omar Bashir, Muhammad Arabi}

King Abdul Aziz Medical City, National Guards Health Affairs Riyadh, Riyadh, Kingdom of Saudi Arabia.

E-mail: turkihamdi164@gmail.com

Background: This retrospective study aims to evaluate the outcome of microwave ablation (Emprint, Medtronics) for managing early stage hepatocellular carcinoma (HCC) at our institution. Methods: On retrospective review of our computerized database, 13 microwave ablation procedures for 11 patients were identified from December 2015 to December 2016. Patient demographics, serology, Child score, ECOG performance status, tumor characteristics and technical parameters were reviewed. All procedures were performed under conscious sedation or general anesthesia. Tumor response was assessed on follow-up cross-sectional imaging. Results: 11 patients (5 female and 6 male) with an age range of 37 years to 72 years with a mean age of 53 years were identified. Most patients demonstrated Child score A and were suffering from either hepatitis B or C.
Mean diameter of the lesion was $2 \mathrm{~cm} .4$ treated lesions were sub-capsular in location whereas the others were parenchymal or centrally located. Most the procedures were performed under both ultrasound and CT guidance. We used a 13 gauge microwave antenna at 100 watts with a mean ablation of time duration of 2.8 minutes. Initial cross-sectional imaging follow-up was performed at a mean interval of 24 days. 8 patients showed complete response after the first session. 3 patients required additional procedures including repeat ablation as well as radiotherapy. One patient developed a small sympathetic pleural effusion after the procedure. No other complications were noted. Conclusions: Our initial results show microwave ablation an effective and safe therapeutic option in the management of early stage HCC.

\section{OC 2.3}

To Determine the Health-related Quality of Life in Patients Undergoing Transarterial Chemoembolization for Hepatocellular Carcinoma

\section{Nauman Al-Qamari, Hatem Adel, Syed Omair Adil, Amjad Sattar}

Dow University of Health Sciences, Karachi, Pakistan

E-mail: naumanalqamari@gmail.com

Background: Liver cancer is third common cause of death worldwide. Hepatocellular carcinoma (HCC) is the most common liver cancer. Majority of cases are detected at advanced stage of disease. $<50 \%$ patients are treated with transarterial chemoembolization (TACE), radiofrequency ablation (RFA) and percutaneous ethanol injection (PEI). TACE is considered as palliative treatment which increases the patients 3 years survival by $40 \%$ but it is associated with many significant complications including postembolization syndrome and chemotherapy related symptoms. Most of the physicians only focus on disease outcome, although patient's quality of life (QOL) has significant impact on disease outcome. To our knowledge, overall few studies has been conducted to see patient's QOL after TACE, but none in our region. Hence this was rational for our study. Methods: Before and after survey was conducted at Vascular Interventional Radiology (VIR) department of DUHS from Feb 2014 to Feb 2015. All patients who underwent TACE irrespective of age and gender with at least one follow up at or after 06 weeks were included. Patients who lost to follow up were excluded. SF 12 questionnaire was used to assess health-related quality of life before and after TACE. Patient demographics like age, gender, follow-up duration, bilirubin level, tumor size and Child Pugh score was calculated. All quantitative variables were presented in the form of median and interquartile range. Wilcoxon sign rank test was applied to see the difference before and after TACE. $P$ value $<0.05$ was taken as significant. Results: Out of total 53 patients, female preponderance was found to be higher $42(79.2 \%)$ than that of males 11 (20.8\%). Majority of the patients were had hepatitis C 47 (88.7\%) whereas hepatitis $\mathrm{B}$ infection was found in $6(11.3 \%)$ patients. Majority of the patients were presented with child score B 34 (64.2\%) followed by child score A 17 (32.1\%) whereas only 2 (3.8\%) patients were presented with child score C. Significant difference was observed in the physical and mental quality of life before and after TACE, i.e. $45.3(42.2-47)$ vs. $46.2(44.2-47.2), P$ value $<0.001$ and 52.3 (48.9-54) vs. 56.3 (53.4-58.9), $P$ value $<0.001$ respectively. 
Conclusions: Significant improvement in physical and mental health related quality of life was observed in patients suffering from hepatocellular carcinoma undergoing TACE.

OC 2.4

Five-year Experience of Percutaneous Cryoablation of Symptomatic Venous Vascular Malformations as Second-Line Therapeutic Option

\section{Francois H. Cornelis, Francois Marin ${ }^{1}$,} Christine Labreze ${ }^{1}$, Nicolas Grenier ${ }^{1}$

Tenon Hospital, Paris, 'Pellegrin Hospital, Bordeaux, France.

E-mail: francoiscornelis@hotmail.com

Background: To report the mid-term outcomes of percutaneous cryoablation (PCA) performed as second-line therapeutic option of venous vascular malformations (VVM). Methods: From 2011 to 2015, PCA was offered in 24 patients (mean age: 31 years, range: 12-64) as second-line treatment for recurrences of symptoms after sclerotherapy and when resection was not possible (due to lesion location or previous failure) or refused by the patient. Adverse effects were recorded, disease-free survival (DFS) and local tissue control (LTC) rates were calculated based on symptoms and volume evolution. Results: Mean follow-up was 18.7 months (6-48). Nine $(37.5 \%, 9 / 24)$ adverse effects occurred but only three $(12.5 \%, 3 / 24)$ were severe. Mean pain assessed by visual analogic scale (VAS) was $41.7 \mathrm{~mm}(0-80)$ before treatment and $20.3 \mathrm{~mm}(0-80)(P=0.01)$ after. Mean volume decreased significantly after treatment from $22.4 \mathrm{~cm} 3(0.9-146)$ to 8.35 cm3 $(0-81.3)(P<0.001)$. Pain recurred in nine patients and size of one lesion increased. The DFS and LTC rates were $54 \%$ [95\% CI: 22.94-77.27] and 93.33\% [61.26-99.03] at 24 months, respectively. Only VVM volume $>10 \mathrm{~cm}^{3}$ was associated with a higher risk of local recurrence $(P=0.05)$. Conclusions: PCA as second-line treatment appears to be safe and effective for local control of VVM according to mid-term results.

\section{OC 2.5}

Bedside Intravascular Ultrasound-guided
Inferior Vena Cava Filter Placement in
Critically-Ill Patients
Mohammad Abusedera, Kyung Cho',
David Williams

Sohag University, Sohag, Egypt, ' University of Michigan, Ann Arbor, Michigan, United States of America.

E-mail:malaa2@yahoo.com

Background: Pulmonary Embolism (PE) is a potentially life-threatening complication of critical illness. Prophylactic inferior vena cava filter (IVC) placement offers a protection rate of $99 \%$ against fatal PE. Methods: Bedside IVC filter insertion guided by IVUS in 37 consecutive critically-ill patients. All patients had clinical indications for IVC interruption; including prophylaxis in high risk patient in the absence of DVT or $P E(n=27)$. The other indication was that patients were suffering from PE and/or DVT with a contraindication to anticoagulation, or ineffective anticoagulation. Transportation to angio- suite was risky or not feasible. Results: 37 patients, 13 patients were female and 24 were male with age ranged from 18 to 80 years with an average age of 44 years old. The filters were placed correctly in 35 of 37 patients $(95 \%)$. filter was inserted at iliac vein in 2 patients that were retrieved and IVC filter was inserted guided by Fluorsocopy via transgugular approach. Placement timing was 5 days at average for surgical ICU patient compared to 17 days for medical ICU patients. infrarenal IVC diameter was $21.8 \mathrm{~mm}$. There were no filter-related complications such as migration penetration or Filter-related thrombosis. There was no recorded incident of PE after IVC filter deployment. Only one filter was retrieved 20 days after placement. Conclusions: Bedside IVUS guided IVC filter placement for critically ill patient in the intensive care unit is a radiation free, contrast free and it is not limited by patient's obesity or recent abdominal surgeries or orthopedic hardware which could be limiting factor for trans abdominal duplex Doppler guided technique.

\section{OC 2.6 (First place oral presentation prize winner) \\ Day Case Endovascular Aneurysm Repair - Our Experience after 250 Patients}

\section{Ahmed Kaabneh}

James Cook University Hospital, Middlesbrough, United Kingdom.

E-mail: ahmedkaabneh@icloud.com

Background: With more than 1000 EVAR patients experience in our centre, the advancement of ultra-low profile EVAR devices and percutaneous access, discharging uncomplicated EVARs in less than 24 hours is becoming more common. Methods: Single centre retrospective analysis of prospectively gathered data on 250 consecutive elective day-case EVAR cases (dEVAR). Patients for dEVAR are selected following joint radiology, surgical and anaesthetic team meeting using UK day-case surgery and locally agreed guidance. Patients deemed suitable are put on a special dEVAR pathway to be admitted on day of surgery and discharged in less than 24 hours. Results: 220 patients were followed-up after dEVAR. 98\% were successfully discharged in less than $24 \mathrm{hrs}$ post-operatively. One patient $(0.45 \%)$ with access vessel complications required additional procedures and had to be hospitalised for two days. One patients $(0.45 \%)$ with non-cardiac chest pain was hospitalised for two days. Two patients (1\%) failed to be discharged within 24 hours but no clear cause documented in the notes. None of the dEVAR patients had a re-admission to hospital within 30 days with no 30-day mortality. Cost comparison showed dEVAR led to reduced overall average cost when compared to standard EVAR from $\leq 13,705$ (CI $= \pm 685$ ) to $\leq 9,330(\mathrm{CI}= \pm 735)$. Conclusions: dEVAR is not for every patient but can be performed safely under appropriate criteria. In this series morbidity was minimal with significant cost saving.

P101

Interventional Radiology: Giving Cosmetic Medicine a Makeover

\section{Samantha Huq, Erik Bee ${ }^{1}$}

University of Connecticut School of Medicine, Farmington, ' $S$ t. Francis Hospital and Medical Center, Hartford, United States of America. E-mail: shuq@uchc.edu

Background: The global cosmetic market has been on the rise with a recent surge in minimally invasive procedures. Cosmetic 